\title{
JMASM38: Confidence Intervals for Kendall's Tau with Small Samples (SPSS)
}

David A. Walker

Northern Illinois University, dawalker@niu.edu

Follow this and additional works at: http://digitalcommons.wayne.edu/jmasm

Part of the Applied Statistics Commons, Social and Behavioral Sciences Commons, and the Statistical Theory Commons

\section{Recommended Citation}

Walker, David A. (2016) "JMASM38: Confidence Intervals for Kendall's Tau with Small Samples (SPSS)," Journal of Modern Applied Statistical Methods: Vol. 15 : Iss. 1, Article 45.

DOI: $10.22237 /$ jmasm/1462077840

Available at: http://digitalcommons.wayne.edu/jmasm/vol15/iss1/45

This Algorithms and Code is brought to you for free and open access by the Open Access Journals at DigitalCommons@WayneState. It has been accepted for inclusion in Journal of Modern Applied Statistical Methods by an authorized editor of DigitalCommons@WayneState. 


\section{JMASM38: Confidence Intervals for Kendall's Tau with Small Samples (SPSS)}

\section{Erratum}

This paper was originally published in JMASM Algorithms \& Code without its enumeration, JMASM38. 


\section{JMASM Algorithms and Code Confidence Intervals for Kendall's Tau with Small Samples}

\section{David A. Walker}

Northern Illinois University

DeKalb, IL

A syntax program, not readily expedient in statistical software such as SPSS, is provided for an application of confidence interval estimates with Kendall's tau-b for small samples.

Keywords: $\quad$ Kendall's tau, confidence intervals, SPSS, syntax

\section{Introduction}

This syntax program (Walker, 2015) is intended to provide an application, not readily available, for users in SPSS who are interested in a range of confidence interval (CI) estimates with Kendall's tau-b $(\tau)$ for small samples. Statistically, Kendall's tau-b is a non-parametric, correlational method typically employed with ordinal $x$ and $y$ measures. Tau's application within social science research has been predominantly in correlational meta-analysis studies and also as a component of experimental research (Cooper \& Hedges, 1994; Gilpin, 1993). Furthermore, tau has been highlighted as a proxy for Pearson's product-moment correlation $(r)$ in research situations where sample sizes are small (Rupinski \& Dunlap, 1996). Tau is expressed as (Helsel \& Hirsch, 1995):

$$
\tau=\frac{C-D}{N(N-1) / 2}
$$

where $C=$ number of concordant pairs, $D=$ number of discordant pairs, and $N=$ sample size.

Dr. Walker is a Professor of Educational Research and Assessment. Email him at: dawalker@niu.edu. 


\section{DAVID A. WALKER}

According to Bonett and Wright (2000), "interval estimation may be more appropriate in applications where the magnitude of a correlation is of primary interest" (p. 23). Certainly, there has been prominence afforded in the literature to this issue that confidence intervals need to be reported with point estimates, such as tau, to supplement and progress the interpretation of outcomes (American Psychological Association, 2010; Levin \& Robinson, 2003).

Further, Long and Cliff (1997), supposing a bivariate normal population, found that tau performed reasonably well with small samples $>10$ and $<25$, where samples $\geq 25$ could be conducted with a Pearson $r$ correlation because of $x$ and $y$ 's sampling from a bivariate normal distribution (Kendall, 1949). Lower and upper bound confidence intervals for tau can be represented through a series of steps such that Fisher's $z$-transformation is used for "normalizing the sampling distribution of $\tau$ ” (Long \& Cliff, 1997, p. 35). Specifically,

$$
\tau_{z}=0.5 \ln \left(\frac{1+\tau}{1-\tau}\right)
$$

Per Fieller, Hartley, and Pearson (1957), the standard error of $\tau_{z}\left[\operatorname{SE}\left(\tau_{z}\right)\right]$ is used with the desired unit normal critical value to construct the lower and upper confidence bounds for $\tau_{z}$ :

$$
\tau_{z . \text { lower }}=\tau_{z}-z_{1-\alpha} \operatorname{SE}\left(\tau_{z}\right)
$$

and

$$
\tau_{z \text {.upper }}=\tau_{z}+z_{1-\alpha} \operatorname{SE}\left(\tau_{z}\right)
$$

where $\tau_{z \text {.lower }}$ and $\tau_{z \text {.upper }}$ are the lower and upper bounds, respectively; $z_{1-\alpha}$ is the unit normal critical $z$ value for specified level of confidence $1-\alpha$; and

$$
\operatorname{SE}\left(\tau_{z}\right)=\sqrt{\frac{0.437}{N-4}}
$$

The values of the previously-mentioned lower and upper confidence bounds for $\tau_{z}$ are then transformed (Fisher, 1925) due to tau's ability “....to generalize from the sample to the population correlation for any monotonic transformation of bivariate normal variables" (Bonett \& Wright, 2000, p. 24). Specifically, 


$$
\tau_{\text {lower }}=\frac{\exp \left(2 \tau_{\text {lower }}\right)-1}{\exp \left(2 \tau_{\text {lower }}\right)+1}
$$

and

$$
\tau_{\text {upper }}=\frac{\exp \left(2 \tau_{\text {upper }}\right)-1}{\exp \left(2 \tau_{\text {upper }}\right)+1}
$$

\section{Confidence Intervals for Kendall's Tau with Small Samples Program}

The independent SPSS syntax platform allows the user to merely run the program, which yields a comprehensive list of tau values ranging from 0.99 to -0.99 , in increments of 0.01 , for small samples extending from 11 to 24 , per the Long and Cliff (1997) recommendation. The confidence intervals presented in conjunction with the tau values are programmed at $80 \%, 90 \%, 95 \%$, and $99 \%$, where Cohen (1990, p. 1310) observed "I don't think that we should routinely use 95\% intervals: Our interests are often better served by more tolerant $80 \%$ intervals." Further, Tukey (1960) added that the application of confidence intervals was of paramount importance,

Probably the greatest ultimate importance, among all types of statistical procedures we now know, belongs to confidence procedures which, by making interval estimates, attempt to reach as strong conclusions as are reasonable by pointing out, not single likely values, but rather whole classes (intervals, regions, etc.) of possible values, so chosen that there can be high confidence that the "true" value is somewhere among them. (p. 429)

It should be noted that this program produces an exceedingly large table (i.e., Table 1 is over 60 pages) and; therefore, only a sample of the results are shown below. The selection of results display an assortment of magnitude and directionality with tau and small samples from 11 to 24 . The array of example tau values include positive, large tau ranging from 0.90 to 0.99 ; positive, moderate values from 0.40 to 0.50 ; and negative, small values extending from -0.20 to -0.10 , all with accompanying CIs. 


\section{DAVID A. WALKER}

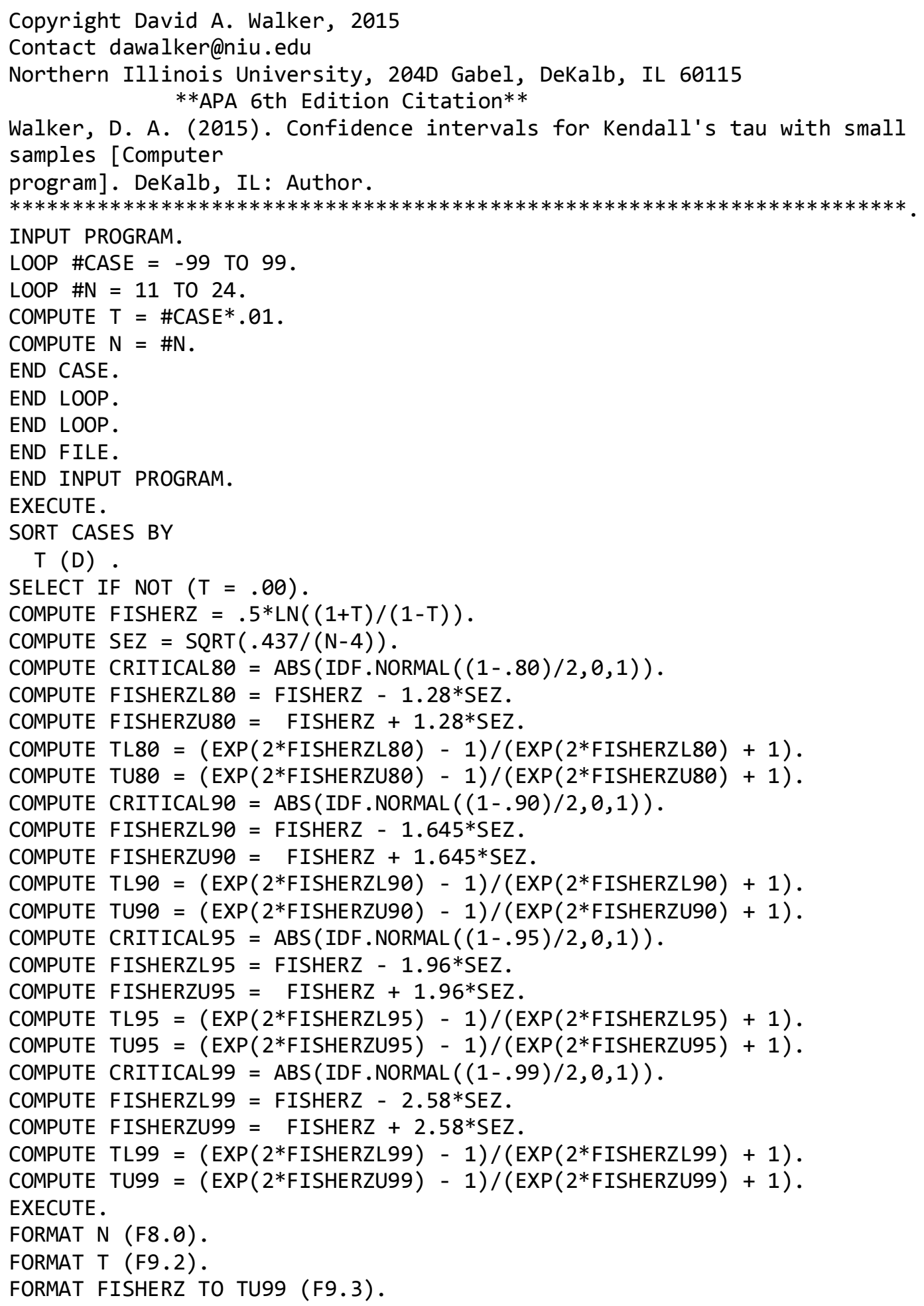




\title{
CONFIDENCE INTERVALS FOR KENDALL'S TAU
}

\author{
VARIABLE LABELS T 'Tau' /TU90 'Upper 90\% CI'/TL90 'Lower 90\% CI'/TU95 \\ 'Upper 95\% CI'/TL95 'Lower 95\% CI'/TU80 'Upper 80\% CI'/TL80 'Lower 80\% \\ CI'/TU99 'Upper 99\% CI'/TL99 'Lower 99\% CI'. \\ REPORT FORMAT=LIST AUTOMATIC ALIGN (CENTER) \\ /VARIABLES $=$ N T TL99 TL95 TL90 TL80 TU80 TU90 TU95 TU99
}

/TITLE "Sample Size, Kendall's Tau, and 80\%, 90\%, 95\%, 99\% Confidence Intervals".

Table 1. Sample size, Kendall's tau, and confidence intervals

\begin{tabular}{|c|c|c|c|c|c|c|c|c|c|}
\hline$N$ & Tau & L $99 \% \mathrm{Cl}$ & L $95 \% \mathrm{Cl}$ & L $90 \% \mathrm{Cl}$ & L $80 \% \mathrm{Cl}$ & U $80 \% \mathrm{Cl}$ & U $90 \% \mathrm{Ci}$ & U 95\% $\mathrm{Cl}$ & U $99 \% \mathrm{Cl}$ \\
\hline 11 & 0.99 & 0.964 & 0.974 & 0.977 & 0.981 & 0.995 & 0.996 & 0.996 & 0.997 \\
\hline 12 & 0.99 & 0.967 & 0.975 & 0.979 & 0.982 & 0.994 & 0.995 & 0.996 & 0.997 \\
\hline 13 & 0.99 & 0.969 & 0.976 & 0.979 & 0.982 & 0.994 & 0.995 & 0.996 & 0.997 \\
\hline 14 & 0.99 & 0.971 & 0.977 & 0.980 & 0.983 & 0.994 & 0.995 & 0.996 & 0.997 \\
\hline 15 & 0.99 & 0.972 & 0.978 & 0.981 & 0.983 & 0.994 & 0.995 & 0.995 & 0.996 \\
\hline 16 & 0.99 & 0.973 & 0.979 & 0.981 & 0.984 & 0.994 & 0.995 & 0.995 & 0.996 \\
\hline 17 & 0.99 & 0.974 & 0.980 & 0.982 & 0.984 & 0.994 & 0.995 & 0.995 & 0.996 \\
\hline 18 & 0.99 & 0.975 & 0.980 & 0.982 & 0.984 & 0.994 & 0.994 & 0.995 & 0.996 \\
\hline 19 & 0.99 & 0.976 & 0.981 & 0.983 & 0.985 & 0.994 & 0.994 & 0.995 & 0.996 \\
\hline 20 & 0.99 & 0.977 & 0.981 & 0.983 & 0.985 & 0.993 & 0.994 & 0.995 & 0.996 \\
\hline 21 & 0.99 & 0.977 & 0.981 & 0.983 & 0.985 & 0.993 & 0.994 & 0.995 & 0.996 \\
\hline 22 & 0.99 & 0.978 & 0.982 & 0.983 & 0.985 & 0.993 & 0.994 & 0.995 & 0.996 \\
\hline 23 & 0.99 & 0.978 & 0.982 & 0.984 & 0.985 & 0.993 & 0.994 & 0.994 & 0.995 \\
\hline 24 & 0.99 & 0.979 & 0.982 & 0.984 & 0.985 & 0.993 & 0.994 & 0.994 & 0.995 \\
\hline 11 & 0.98 & 0.929 & 0.948 & 0.955 & 0.962 & 0.989 & 0.991 & 0.992 & 0.994 \\
\hline 12 & 0.98 & 0.935 & 0.951 & 0.957 & 0.964 & 0.989 & 0.991 & 0.992 & 0.994 \\
\hline 13 & 0.98 & 0.939 & 0.953 & 0.959 & 0.965 & 0.989 & 0.990 & 0.992 & 0.994 \\
\hline 14 & 0.98 & 0.942 & 0.955 & 0.961 & 0.966 & 0.988 & 0.990 & 0.991 & 0.993 \\
\hline 15 & 0.98 & 0.945 & 0.957 & 0.962 & 0.967 & 0.988 & 0.990 & 0.991 & 0.993 \\
\hline 16 & 0.98 & 0.947 & 0.958 & 0.963 & 0.968 & 0.988 & 0.989 & 0.990 & 0.992 \\
\hline 17 & 0.98 & 0.949 & 0.959 & 0.964 & 0.968 & 0.987 & 0.989 & 0.990 & 0.992 \\
\hline 18 & 0.98 & 0.951 & 0.960 & 0.965 & 0.969 & 0.987 & 0.989 & 0.990 & 0.992 \\
\hline 19 & 0.98 & 0.952 & 0.961 & 0.965 & 0.969 & 0.987 & 0.989 & 0.990 & 0.992 \\
\hline 20 & 0.98 & 0.954 & 0.962 & 0.966 & 0.970 & 0.987 & 0.988 & 0.989 & 0.991 \\
\hline 21 & 0.98 & 0.955 & 0.963 & 0.966 & 0.970 & 0.987 & 0.988 & 0.989 & 0.991 \\
\hline 22 & 0.98 & 0.956 & 0.963 & 0.967 & 0.970 & 0.987 & 0.988 & 0.989 & 0.991 \\
\hline 23 & 0.98 & 0.957 & 0.964 & 0.967 & 0.971 & 0.986 & 0.988 & 0.989 & 0.991 \\
\hline 24 & 0.98 & 0.958 & 0.965 & 0.968 & 0.971 & 0.986 & 0.988 & 0.989 & 0.991 \\
\hline 11 & 0.97 & 0.895 & 0.922 & 0.933 & 0.944 & 0.984 & 0.987 & 0.989 & 0.992 \\
\hline 12 & 0.97 & 0.903 & 0.927 & 0.936 & 0.946 & 0.983 & 0.986 & 0.988 & 0.991 \\
\hline 13 & 0.97 & 0.909 & 0.930 & 0.939 & 0.948 & 0.983 & 0.985 & 0.987 & 0.990 \\
\hline 14 & 0.97 & 0.914 & 0.933 & 0.941 & 0.949 & 0.982 & 0.985 & 0.987 & 0.990 \\
\hline 15 & 0.97 & 0.918 & 0.936 & 0.943 & 0.951 & 0.982 & 0.984 & 0.986 & 0.989 \\
\hline 16 & 0.97 & 0.922 & 0.938 & 0.945 & 0.952 & 0.981 & 0.984 & 0.986 & 0.989 \\
\hline 17 & 0.97 & 0.925 & 0.939 & 0.946 & 0.952 & 0.981 & 0.983 & 0.985 & 0.988 \\
\hline
\end{tabular}


DAVID A. WALKER

Table 1, continued.

\begin{tabular}{|c|c|c|c|c|c|c|c|c|c|}
\hline$N$ & Tau & L 99\% Cl & L 95\% Cl & L $90 \% \mathrm{Cl}$ & $\mathrm{L} 80 \% \mathrm{Cl}$ & $\mathrm{U} 80 \% \mathrm{Cl}$ & U $90 \% \mathrm{Ci}$ & U 95\% Cl & U $99 \% \mathrm{Cl}$ \\
\hline 18 & 0.97 & 0.927 & 0.941 & 0.947 & 0.953 & 0.981 & 0.983 & 0.985 & 0.988 \\
\hline 19 & 0.97 & 0.929 & 0.942 & 0.948 & 0.954 & 0.981 & 0.983 & 0.985 & 0.987 \\
\hline 20 & 0.97 & 0.931 & 0.943 & 0.949 & 0.955 & 0.980 & 0.982 & 0.984 & 0.987 \\
\hline 21 & 0.97 & 0.933 & 0.944 & 0.950 & 0.955 & 0.980 & 0.982 & 0.984 & 0.987 \\
\hline 22 & 0.97 & 0.934 & 0.945 & 0.950 & 0.956 & 0.980 & 0.982 & 0.984 & 0.986 \\
\hline 23 & 0.97 & 0.936 & 0.946 & 0.951 & 0.956 & 0.980 & 0.982 & 0.983 & 0.986 \\
\hline 24 & 0.97 & 0.937 & 0.947 & 0.952 & 0.957 & 0.979 & 0.981 & 0.983 & 0.986 \\
\hline 11 & 0.96 & 0.862 & 0.897 & 0.911 & 0.926 & 0.979 & 0.982 & 0.985 & 0.989 \\
\hline 12 & 0.96 & 0.872 & 0.903 & 0.916 & 0.928 & 0.978 & 0.981 & 0.984 & 0.988 \\
\hline 13 & 0.96 & 0.880 & 0.908 & 0.919 & 0.931 & 0.977 & 0.980 & 0.983 & 0.987 \\
\hline 14 & 0.96 & 0.887 & 0.911 & 0.922 & 0.933 & 0.976 & 0.980 & 0.982 & 0.986 \\
\hline 15 & 0.96 & 0.892 & 0.915 & 0.924 & 0.934 & 0.976 & 0.979 & 0.981 & 0.986 \\
\hline 16 & 0.96 & 0.896 & 0.917 & 0.926 & 0.936 & 0.975 & 0.978 & 0.981 & 0.985 \\
\hline 17 & 0.96 & 0.900 & 0.920 & 0.928 & 0.937 & 0.975 & 0.978 & 0.980 & 0.984 \\
\hline 18 & 0.96 & 0.903 & 0.922 & 0.930 & 0.938 & 0.974 & 0.977 & 0.980 & 0.984 \\
\hline 19 & 0.96 & 0.906 & 0.923 & 0.931 & 0.939 & 0.974 & 0.977 & 0.979 & 0.983 \\
\hline 20 & 0.96 & 0.909 & 0.925 & 0.932 & 0.940 & 0.974 & 0.977 & 0.979 & 0.983 \\
\hline 21 & 0.96 & 0.911 & 0.926 & 0.933 & 0.940 & 0.973 & 0.976 & 0.978 & 0.982 \\
\hline 22 & 0.96 & 0.913 & 0.928 & 0.934 & 0.941 & 0.973 & 0.976 & 0.978 & 0.982 \\
\hline 23 & 0.96 & 0.915 & 0.929 & 0.935 & 0.942 & 0.973 & 0.976 & 0.978 & 0.982 \\
\hline 24 & 0.96 & 0.916 & 0.930 & 0.936 & 0.942 & 0.972 & 0.975 & 0.977 & 0.981 \\
\hline 11 & 0.95 & 0.830 & 0.872 & 0.890 & 0.907 & 0.973 & 0.978 & 0.981 & 0.986 \\
\hline 12 & 0.95 & 0.842 & 0.880 & 0.895 & 0.911 & 0.972 & 0.977 & 0.980 & 0.985 \\
\hline 13 & 0.95 & 0.852 & 0.885 & 0.899 & 0.914 & 0.971 & 0.975 & 0.979 & 0.984 \\
\hline 14 & 0.95 & 0.860 & 0.890 & 0.903 & 0.916 & 0.970 & 0.975 & 0.978 & 0.983 \\
\hline 15 & 0.95 & 0.866 & 0.894 & 0.906 & 0.918 & 0.970 & 0.974 & 0.977 & 0.982 \\
\hline 16 & 0.95 & 0.872 & 0.897 & 0.908 & 0.920 & 0.969 & 0.973 & 0.976 & 0.981 \\
\hline 17 & 0.95 & 0.876 & 0.900 & 0.910 & 0.921 & 0.968 & 0.972 & 0.975 & 0.980 \\
\hline 18 & 0.95 & 0.880 & 0.902 & 0.912 & 0.923 & 0.968 & 0.972 & 0.975 & 0.980 \\
\hline 19 & 0.95 & 0.883 & 0.905 & 0.914 & 0.924 & 0.967 & 0.971 & 0.974 & 0.979 \\
\hline 20 & 0.95 & 0.887 & 0.907 & 0.915 & 0.925 & 0.967 & 0.971 & 0.974 & 0.978 \\
\hline 21 & 0.95 & 0.889 & 0.908 & 0.917 & 0.926 & 0.967 & 0.970 & 0.973 & 0.978 \\
\hline 22 & 0.95 & 0.892 & 0.910 & 0.918 & 0.926 & 0.966 & 0.970 & 0.973 & 0.977 \\
\hline 23 & 0.95 & 0.894 & 0.911 & 0.919 & 0.927 & 0.966 & 0.969 & 0.972 & 0.977 \\
\hline 24 & 0.95 & 0.896 & 0.912 & 0.920 & 0.928 & 0.965 & 0.969 & 0.972 & 0.976 \\
\hline 11 & 0.94 & 0.798 & 0.848 & 0.869 & 0.889 & 0.968 & 0.973 & 0.977 & 0.983 \\
\hline 12 & 0.94 & 0.813 & 0.856 & 0.875 & 0.893 & 0.967 & 0.972 & 0.976 & 0.982 \\
\hline 13 & 0.94 & 0.824 & 0.863 & 0.880 & 0.897 & 0.965 & 0.970 & 0.974 & 0.980 \\
\hline 14 & 0.94 & 0.833 & 0.869 & 0.884 & 0.900 & 0.964 & 0.969 & 0.973 & 0.979 \\
\hline 15 & 0.94 & 0.841 & 0.873 & 0.888 & 0.902 & 0.964 & 0.968 & 0.972 & 0.978 \\
\hline 16 & 0.94 & 0.847 & 0.877 & 0.890 & 0.904 & 0.963 & 0.968 & 0.971 & 0.977 \\
\hline 18 & 0.94 & 0.857 & 0.884 & 0.895 & 0.907 & 0.961 & 0.966 & 0.970 & 0.975 \\
\hline 19 & 0.94 & 0.861 & 0.886 & 0.897 & 0.909 & 0.961 & 0.965 & 0.969 & 0.975 \\
\hline 20 & 0.94 & 0.865 & 0.888 & 0.899 & 0.910 & 0.960 & 0.965 & 0.968 & 0.974 \\
\hline 21 & 0.94 & 0.868 & 0.890 & 0.900 & 0.911 & 0.960 & 0.964 & 0.968 & 0.973 \\
\hline
\end{tabular}


CONFIDENCE INTERVALS FOR KENDALL'S TAU

Table 1, continued.

\begin{tabular}{|c|c|c|c|c|c|c|c|c|c|}
\hline$N$ & Tau & L 99\% Cl & L 95\% Cl & L $90 \% \mathrm{Cl}$ & $\mathrm{L} 80 \% \mathrm{Cl}$ & $\mathrm{U} 80 \% \mathrm{Cl}$ & U $90 \% \mathrm{Ci}$ & U 95\% Cl & $\mathrm{U} 99 \% \mathrm{Cl}$ \\
\hline 22 & 0.94 & 0.871 & 0.892 & 0.902 & 0.912 & 0.959 & 0.964 & 0.967 & 0.973 \\
\hline 23 & 0.94 & 0.873 & 0.894 & 0.903 & 0.913 & 0.959 & 0.963 & 0.966 & 0.972 \\
\hline 24 & 0.94 & 0.876 & 0.895 & 0.904 & 0.914 & 0.959 & 0.963 & 0.966 & 0.972 \\
\hline 11 & 0.93 & 0.767 & 0.824 & 0.848 & 0.871 & 0.962 & 0.969 & 0.973 & 0.980 \\
\hline 12 & 0.93 & 0.784 & 0.834 & 0.855 & 0.876 & 0.961 & 0.967 & 0.971 & 0.979 \\
\hline 13 & 0.93 & 0.797 & 0.842 & 0.861 & 0.880 & 0.960 & 0.965 & 0.970 & 0.977 \\
\hline 14 & 0.93 & 0.807 & 0.848 & 0.865 & 0.883 & 0.958 & 0.964 & 0.969 & 0.976 \\
\hline 15 & 0.93 & 0.816 & 0.853 & 0.869 & 0.886 & 0.957 & 0.963 & 0.967 & 0.974 \\
\hline 16 & 0.93 & 0.823 & 0.858 & 0.873 & 0.888 & 0.956 & 0.962 & 0.966 & 0.973 \\
\hline 17 & 0.93 & 0.829 & 0.861 & 0.876 & 0.890 & 0.956 & 0.961 & 0.965 & 0.972 \\
\hline 18 & 0.93 & 0.834 & 0.865 & 0.878 & 0.892 & 0.955 & 0.960 & 0.964 & 0.971 \\
\hline 19 & 0.93 & 0.839 & 0.868 & 0.880 & 0.894 & 0.954 & 0.959 & 0.964 & 0.970 \\
\hline 20 & 0.93 & 0.843 & 0.870 & 0.882 & 0.895 & 0.954 & 0.959 & 0.963 & 0.970 \\
\hline 21 & 0.93 & 0.847 & 0.873 & 0.884 & 0.896 & 0.953 & 0.958 & 0.962 & 0.969 \\
\hline 22 & 0.93 & 0.850 & 0.875 & 0.886 & 0.897 & 0.952 & 0.957 & 0.961 & 0.968 \\
\hline 23 & 0.93 & 0.853 & 0.877 & 0.887 & 0.898 & 0.952 & 0.957 & 0.961 & 0.967 \\
\hline 24 & 0.93 & 0.856 & 0.878 & 0.889 & 0.899 & 0.952 & 0.956 & 0.960 & 0.967 \\
\hline 11 & 0.92 & 0.737 & 0.800 & 0.827 & 0.854 & 0.957 & 0.964 & 0.969 & 0.977 \\
\hline 12 & 0.92 & 0.756 & 0.811 & 0.835 & 0.859 & 0.955 & 0.962 & 0.967 & 0.975 \\
\hline 13 & 0.92 & 0.770 & 0.820 & 0.842 & 0.864 & 0.954 & 0.960 & 0.965 & 0.974 \\
\hline 14 & 0.92 & 0.782 & 0.827 & 0.847 & 0.867 & 0.952 & 0.959 & 0.964 & 0.972 \\
\hline 15 & 0.92 & 0.791 & 0.833 & 0.851 & 0.870 & 0.951 & 0.958 & 0.963 & 0.971 \\
\hline 16 & 0.92 & 0.799 & 0.838 & 0.855 & 0.873 & 0.950 & 0.956 & 0.961 & 0.969 \\
\hline 17 & 0.92 & 0.806 & 0.842 & 0.858 & 0.875 & 0.949 & 0.955 & 0.960 & 0.968 \\
\hline 18 & 0.92 & 0.812 & 0.846 & 0.861 & 0.877 & 0.948 & 0.954 & 0.959 & 0.967 \\
\hline 19 & 0.92 & 0.817 & 0.850 & 0.864 & 0.879 & 0.948 & 0.954 & 0.958 & 0.966 \\
\hline 20 & 0.92 & 0.822 & 0.852 & 0.866 & 0.880 & 0.947 & 0.953 & 0.957 & 0.965 \\
\hline 21 & 0.92 & 0.826 & 0.855 & 0.868 & 0.882 & 0.946 & 0.952 & 0.957 & 0.964 \\
\hline 22 & 0.92 & 0.830 & 0.857 & 0.870 & 0.883 & 0.946 & 0.951 & 0.956 & 0.963 \\
\hline 23 & 0.92 & 0.833 & 0.860 & 0.872 & 0.884 & 0.945 & 0.951 & 0.955 & 0.963 \\
\hline 24 & 0.92 & 0.836 & 0.862 & 0.873 & 0.885 & 0.945 & 0.950 & 0.954 & 0.962 \\
\hline 11 & 0.91 & 0.708 & 0.777 & 0.806 & 0.836 & 0.951 & 0.959 & 0.965 & 0.974 \\
\hline 12 & 0.91 & 0.728 & 0.789 & 0.815 & 0.842 & 0.950 & 0.957 & 0.963 & 0.972 \\
\hline 13 & 0.91 & 0.744 & 0.799 & 0.823 & 0.847 & 0.948 & 0.955 & 0.961 & 0.970 \\
\hline 14 & 0.91 & 0.757 & 0.807 & 0.829 & 0.851 & 0.946 & 0.954 & 0.959 & 0.968 \\
\hline 15 & 0.91 & 0.767 & 0.813 & 0.834 & 0.854 & 0.945 & 0.952 & 0.958 & 0.967 \\
\hline 16 & 0.91 & 0.776 & 0.819 & 0.838 & 0.857 & 0.944 & 0.951 & 0.956 & 0.965 \\
\hline 17 & 0.91 & 0.784 & 0.824 & 0.841 & 0.860 & 0.943 & 0.950 & 0.955 & 0.964 \\
\hline 18 & 0.91 & 0.790 & 0.828 & 0.845 & 0.862 & 0.942 & 0.949 & 0.954 & 0.963 \\
\hline 19 & 0.91 & 0.796 & 0.832 & 0.847 & 0.864 & 0.941 & 0.948 & 0.953 & 0.962 \\
\hline 20 & 0.91 & 0.801 & 0.835 & 0.850 & 0.866 & 0.940 & 0.947 & 0.952 & 0.961 \\
\hline 22 & 0.91 & 0.809 & 0.840 & 0.854 & 0.869 & 0.939 & 0.945 & 0.950 & 0.959 \\
\hline 23 & 0.91 & 0.813 & 0.843 & 0.856 & 0.870 & 0.938 & 0.944 & 0.949 & 0.958 \\
\hline 24 & 0.91 & 0.816 & 0.845 & 0.858 & 0.871 & 0.937 & 0.944 & 0.949 & 0.957 \\
\hline 11 & 0.90 & 0.679 & 0.754 & 0.786 & 0.819 & 0.946 & 0.955 & 0.961 & 0.971 \\
\hline
\end{tabular}


DAVID A. WALKER

Table 1, continued.

\begin{tabular}{|c|c|c|c|c|c|c|c|c|c|}
\hline$N$ & Tau & L 99\% Cl & L 95\% Cl & $\mathrm{L} 90 \% \mathrm{Cl}$ & $\mathrm{L} 80 \% \mathrm{Cl}$ & U $80 \% \mathrm{Cl}$ & U $90 \% \mathbf{C i}$ & U 95\% Cl & U 99\% Cl \\
\hline 12 & 0.90 & 0.701 & 0.767 & 0.796 & 0.825 & 0.944 & 0.952 & 0.959 & 0.969 \\
\hline 13 & 0.90 & 0.718 & 0.778 & 0.804 & 0.831 & 0.942 & 0.950 & 0.957 & 0.967 \\
\hline 14 & 0.90 & 0.732 & 0.787 & 0.810 & 0.835 & 0.940 & 0.948 & 0.955 & 0.965 \\
\hline 15 & 0.90 & 0.743 & 0.794 & 0.816 & 0.839 & 0.939 & 0.947 & 0.953 & 0.963 \\
\hline 16 & 0.90 & 0.753 & 0.800 & 0.820 & 0.842 & 0.937 & 0.945 & 0.951 & 0.961 \\
\hline 17 & 0.90 & 0.761 & 0.805 & 0.824 & 0.845 & 0.936 & 0.944 & 0.950 & 0.960 \\
\hline 18 & 0.90 & 0.768 & 0.810 & 0.828 & 0.847 & 0.935 & 0.943 & 0.949 & 0.959 \\
\hline 19 & 0.90 & 0.775 & 0.814 & 0.831 & 0.849 & 0.934 & 0.942 & 0.948 & 0.957 \\
\hline 20 & 0.90 & 0.780 & 0.817 & 0.834 & 0.851 & 0.933 & 0.941 & 0.946 & 0.956 \\
\hline 21 & 0.90 & 0.785 & 0.820 & 0.836 & 0.853 & 0.933 & 0.940 & 0.945 & 0.955 \\
\hline 22 & 0.90 & 0.790 & 0.823 & 0.838 & 0.855 & 0.932 & 0.939 & 0.944 & 0.954 \\
\hline 23 & 0.90 & 0.794 & 0.826 & 0.840 & 0.856 & 0.931 & 0.938 & 0.944 & 0.953 \\
\hline 24 & 0.90 & 0.797 & 0.828 & 0.842 & 0.857 & 0.930 & 0.937 & 0.943 & 0.952 \\
\hline 11 & 0.50 & -0.095 & 0.060 & 0.137 & 0.226 & 0.701 & 0.744 & 0.778 & 0.832 \\
\hline 12 & 0.50 & -0.054 & 0.091 & 0.163 & 0.245 & 0.690 & 0.732 & 0.765 & 0.819 \\
\hline 13 & 0.50 & -0.019 & 0.117 & 0.185 & 0.261 & 0.681 & 0.722 & 0.754 & 0.807 \\
\hline 14 & 0.50 & 0.010 & 0.139 & 0.203 & 0.275 & 0.673 & 0.713 & 0.744 & 0.796 \\
\hline 15 & 0.50 & 0.035 & 0.157 & 0.218 & 0.286 & 0.667 & 0.705 & 0.735 & 0.787 \\
\hline 16 & 0.50 & 0.057 & 0.174 & 0.231 & 0.296 & 0.660 & 0.698 & 0.727 & 0.779 \\
\hline 17 & 0.50 & 0.076 & 0.188 & 0.243 & 0.305 & 0.655 & 0.692 & 0.720 & 0.771 \\
\hline 18 & 0.50 & 0.093 & 0.200 & 0.253 & 0.312 & 0.650 & 0.686 & 0.714 & 0.764 \\
\hline 19 & 0.50 & 0.109 & 0.212 & 0.262 & 0.319 & 0.646 & 0.681 & 0.708 & 0.757 \\
\hline 20 & 0.50 & 0.122 & 0.222 & 0.271 & 0.325 & 0.642 & 0.676 & 0.703 & 0.751 \\
\hline 21 & 0.50 & 0.135 & 0.231 & 0.278 & 0.331 & 0.638 & 0.671 & 0.698 & 0.746 \\
\hline 22 & 0.50 & 0.146 & 0.239 & 0.285 & 0.336 & 0.634 & 0.667 & 0.694 & 0.740 \\
\hline 23 & 0.50 & 0.157 & 0.247 & 0.291 & 0.341 & 0.631 & 0.663 & 0.689 & 0.735 \\
\hline 24 & 0.50 & 0.166 & 0.254 & 0.297 & 0.345 & 0.628 & 0.660 & 0.685 & 0.731 \\
\hline 11 & 0.49 & -0.108 & 0.046 & 0.124 & 0.213 & 0.694 & 0.738 & 0.772 & 0.828 \\
\hline 12 & 0.49 & -0.067 & 0.078 & 0.150 & 0.233 & 0.683 & 0.726 & 0.759 & 0.814 \\
\hline 13 & 0.49 & -0.032 & 0.104 & 0.172 & 0.249 & 0.674 & 0.716 & 0.748 & 0.802 \\
\hline 14 & 0.49 & -0.003 & 0.126 & 0.190 & 0.262 & 0.666 & 0.706 & 0.738 & 0.791 \\
\hline 15 & 0.49 & 0.022 & 0.144 & 0.205 & 0.274 & 0.659 & 0.698 & 0.729 & 0.782 \\
\hline 16 & 0.49 & 0.044 & 0.161 & 0.219 & 0.284 & 0.653 & 0.691 & 0.721 & 0.773 \\
\hline 17 & 0.49 & 0.063 & 0.175 & 0.230 & 0.293 & 0.647 & 0.685 & 0.714 & 0.765 \\
\hline 18 & 0.49 & 0.080 & 0.188 & 0.241 & 0.300 & 0.642 & 0.679 & 0.708 & 0.758 \\
\hline 19 & 0.49 & 0.095 & 0.199 & 0.250 & 0.307 & 0.638 & 0.673 & 0.702 & 0.752 \\
\hline 20 & 0.49 & 0.109 & 0.209 & 0.258 & 0.314 & 0.634 & 0.668 & 0.696 & 0.745 \\
\hline 21 & 0.49 & 0.122 & 0.218 & 0.266 & 0.319 & 0.630 & 0.664 & 0.691 & 0.740 \\
\hline 22 & 0.49 & 0.133 & 0.227 & 0.273 & 0.324 & 0.626 & 0.660 & 0.687 & 0.734 \\
\hline 23 & 0.49 & 0.144 & 0.234 & 0.279 & 0.329 & 0.623 & 0.656 & 0.682 & 0.729 \\
\hline 24 & 0.49 & 0.153 & 0.241 & 0.285 & 0.334 & 0.620 & 0.652 & 0.678 & 0.725 \\
\hline 12 & 0.48 & -0.080 & 0.065 & 0.138 & 0.220 & 0.676 & 0.720 & 0.754 & 0.810 \\
\hline 13 & 0.48 & -0.045 & 0.091 & 0.159 & 0.236 & 0.667 & 0.709 & 0.742 & 0.797 \\
\hline 14 & 0.48 & -0.016 & 0.113 & 0.177 & 0.250 & 0.659 & 0.700 & 0.732 & 0.787 \\
\hline 15 & 0.48 & 0.009 & 0.132 & 0.193 & 0.262 & 0.652 & 0.692 & 0.723 & 0.777 \\
\hline
\end{tabular}


CONFIDENCE INTERVALS FOR KENDALL'S TAU

Table 1, continued.

\begin{tabular}{|c|c|c|c|c|c|c|c|c|c|}
\hline$N$ & Tau & L 99\% Cl & L 95\% Cl & L $90 \% \mathrm{Cl}$ & $\mathrm{L} 80 \% \mathrm{Cl}$ & $\mathrm{U} 80 \% \mathrm{Cl}$ & U $90 \% \mathrm{Ci}$ & U 95\% Cl & $\mathrm{U} 99 \% \mathrm{Cl}$ \\
\hline 16 & 0.48 & 0.031 & 0.148 & 0.206 & 0.272 & 0.645 & 0.684 & 0.715 & 0.768 \\
\hline 17 & 0.48 & 0.050 & 0.162 & 0.218 & 0.281 & 0.640 & 0.678 & 0.708 & 0.760 \\
\hline 18 & 0.48 & 0.067 & 0.175 & 0.228 & 0.288 & 0.635 & 0.672 & 0.701 & 0.753 \\
\hline 19 & 0.48 & 0.082 & 0.186 & 0.238 & 0.295 & 0.630 & 0.666 & 0.695 & 0.746 \\
\hline 20 & 0.48 & 0.096 & 0.196 & 0.246 & 0.302 & 0.626 & 0.661 & 0.689 & 0.739 \\
\hline 21 & 0.48 & 0.109 & 0.206 & 0.254 & 0.307 & 0.622 & 0.657 & 0.684 & 0.734 \\
\hline 22 & 0.48 & 0.120 & 0.214 & 0.261 & 0.313 & 0.618 & 0.652 & 0.680 & 0.728 \\
\hline 23 & 0.48 & 0.131 & 0.222 & 0.267 & 0.317 & 0.615 & 0.648 & 0.675 & 0.723 \\
\hline 24 & 0.48 & 0.141 & 0.229 & 0.273 & 0.322 & 0.612 & 0.645 & 0.671 & 0.718 \\
\hline 11 & 0.47 & -0.134 & 0.020 & 0.099 & 0.188 & 0.680 & 0.726 & 0.762 & 0.819 \\
\hline 12 & 0.47 & -0.093 & 0.052 & 0.125 & 0.208 & 0.669 & 0.714 & 0.748 & 0.805 \\
\hline 13 & 0.47 & -0.058 & 0.078 & 0.147 & 0.224 & 0.660 & 0.703 & 0.736 & 0.793 \\
\hline 14 & 0.47 & -0.029 & 0.100 & 0.165 & 0.238 & 0.651 & 0.693 & 0.726 & 0.782 \\
\hline 15 & 0.47 & -0.004 & 0.119 & 0.180 & 0.250 & 0.644 & 0.685 & 0.717 & 0.772 \\
\hline 16 & 0.47 & 0.018 & 0.135 & 0.194 & 0.260 & 0.638 & 0.677 & 0.708 & 0.763 \\
\hline 17 & 0.47 & 0.037 & 0.150 & 0.205 & 0.269 & 0.632 & 0.671 & 0.701 & 0.754 \\
\hline 18 & 0.47 & 0.054 & 0.162 & 0.216 & 0.277 & 0.627 & 0.664 & 0.694 & 0.747 \\
\hline 19 & 0.47 & 0.070 & 0.174 & 0.225 & 0.284 & 0.622 & 0.659 & 0.688 & 0.740 \\
\hline 20 & 0.47 & 0.083 & 0.184 & 0.234 & 0.290 & 0.618 & 0.654 & 0.683 & 0.734 \\
\hline 21 & 0.47 & 0.096 & 0.193 & 0.241 & 0.296 & 0.614 & 0.649 & 0.677 & 0.728 \\
\hline 22 & 0.47 & 0.108 & 0.202 & 0.248 & 0.301 & 0.610 & 0.645 & 0.673 & 0.722 \\
\hline 23 & 0.47 & 0.118 & 0.210 & 0.255 & 0.306 & 0.607 & 0.641 & 0.668 & 0.717 \\
\hline 24 & 0.47 & 0.128 & 0.217 & 0.261 & 0.310 & 0.604 & 0.637 & 0.664 & 0.712 \\
\hline 11 & 0.46 & -0.146 & 0.008 & 0.086 & 0.176 & 0.674 & 0.720 & 0.756 & 0.815 \\
\hline 12 & 0.46 & -0.105 & 0.039 & 0.112 & 0.196 & 0.662 & 0.707 & 0.742 & 0.801 \\
\hline 13 & 0.46 & -0.071 & 0.065 & 0.134 & 0.212 & 0.652 & 0.696 & 0.730 & 0.788 \\
\hline 14 & 0.46 & -0.042 & 0.087 & 0.152 & 0.226 & 0.644 & 0.686 & 0.720 & 0.777 \\
\hline 15 & 0.46 & -0.017 & 0.106 & 0.168 & 0.238 & 0.637 & 0.678 & 0.710 & 0.766 \\
\hline 16 & 0.46 & 0.005 & 0.123 & 0.181 & 0.248 & 0.630 & 0.670 & 0.702 & 0.757 \\
\hline 17 & 0.46 & 0.024 & 0.137 & 0.193 & 0.257 & 0.624 & 0.663 & 0.695 & 0.749 \\
\hline 18 & 0.46 & 0.041 & 0.150 & 0.204 & 0.265 & 0.619 & 0.657 & 0.688 & 0.741 \\
\hline 19 & 0.46 & 0.057 & 0.161 & 0.213 & 0.272 & 0.614 & 0.652 & 0.681 & 0.734 \\
\hline 20 & 0.46 & 0.071 & 0.172 & 0.222 & 0.278 & 0.610 & 0.646 & 0.676 & 0.728 \\
\hline 21 & 0.46 & 0.083 & 0.181 & 0.229 & 0.284 & 0.606 & 0.642 & 0.670 & 0.722 \\
\hline 22 & 0.46 & 0.095 & 0.190 & 0.236 & 0.289 & 0.602 & 0.637 & 0.666 & 0.716 \\
\hline 23 & 0.46 & 0.106 & 0.197 & 0.243 & 0.294 & 0.599 & 0.633 & 0.661 & 0.711 \\
\hline 24 & 0.46 & 0.115 & 0.205 & 0.249 & 0.299 & 0.596 & 0.629 & 0.657 & 0.706 \\
\hline 11 & 0.45 & -0.159 & -0.005 & 0.074 & 0.163 & 0.667 & 0.714 & 0.751 & 0.811 \\
\hline 12 & 0.45 & -0.118 & 0.027 & 0.100 & 0.183 & 0.655 & 0.701 & 0.737 & 0.796 \\
\hline 13 & 0.45 & -0.084 & 0.053 & 0.122 & 0.200 & 0.645 & 0.690 & 0.724 & 0.783 \\
\hline 14 & 0.45 & -0.055 & 0.075 & 0.140 & 0.214 & 0.637 & 0.680 & 0.714 & 0.772 \\
\hline 16 & 0.45 & -0.008 & 0.110 & 0.169 & 0.236 & 0.622 & 0.663 & 0.696 & 0.752 \\
\hline 17 & 0.45 & 0.012 & 0.125 & 0.181 & 0.245 & 0.617 & 0.656 & 0.688 & 0.743 \\
\hline 18 & 0.45 & 0.029 & 0.138 & 0.192 & 0.253 & 0.611 & 0.650 & 0.681 & 0.735 \\
\hline 19 & 0.45 & 0.044 & 0.149 & 0.201 & 0.260 & 0.606 & 0.644 & 0.675 & 0.728 \\
\hline
\end{tabular}


DAVID A. WALKER

Table 1, continued.

\begin{tabular}{|c|c|c|c|c|c|c|c|c|c|}
\hline$N$ & Tau & L 99\% Cl & L 95\% Cl & $\mathrm{L} 90 \% \mathrm{Cl}$ & $\mathrm{L} 80 \% \mathrm{Cl}$ & U $80 \% \mathrm{Cl}$ & U 90\% Ci & U 95\% Cl & U 99\% Cl \\
\hline 20 & 0.45 & 0.058 & 0.159 & 0.210 & 0.267 & 0.602 & 0.639 & 0.669 & 0.722 \\
\hline 21 & 0.45 & 0.071 & 0.169 & 0.217 & 0.272 & 0.598 & 0.634 & 0.663 & 0.715 \\
\hline 22 & 0.45 & 0.083 & 0.177 & 0.224 & 0.278 & 0.594 & 0.630 & 0.658 & 0.710 \\
\hline 23 & 0.45 & 0.093 & 0.185 & 0.231 & 0.283 & 0.591 & 0.626 & 0.654 & 0.704 \\
\hline 24 & 0.45 & 0.103 & 0.193 & 0.237 & 0.287 & 0.588 & 0.622 & 0.649 & 0.699 \\
\hline 11 & 0.44 & -0.171 & -0.017 & 0.061 & 0.151 & 0.660 & 0.708 & 0.745 & 0.806 \\
\hline 12 & 0.44 & -0.130 & 0.014 & 0.088 & 0.171 & 0.648 & 0.695 & 0.731 & 0.791 \\
\hline 13 & 0.44 & -0.096 & 0.040 & 0.109 & 0.188 & 0.638 & 0.683 & 0.718 & 0.778 \\
\hline 14 & 0.44 & -0.067 & 0.062 & 0.128 & 0.202 & 0.629 & 0.673 & 0.707 & 0.766 \\
\hline 15 & 0.44 & -0.042 & 0.081 & 0.143 & 0.214 & 0.621 & 0.664 & 0.698 & 0.756 \\
\hline 16 & 0.44 & -0.020 & 0.098 & 0.157 & 0.224 & 0.615 & 0.656 & 0.689 & 0.746 \\
\hline 17 & 0.44 & -0.001 & 0.112 & 0.169 & 0.233 & 0.609 & 0.649 & 0.681 & 0.738 \\
\hline 18 & 0.44 & 0.016 & 0.125 & 0.180 & 0.241 & 0.603 & 0.643 & 0.674 & 0.730 \\
\hline 19 & 0.44 & 0.032 & 0.137 & 0.189 & 0.248 & 0.598 & 0.637 & 0.668 & 0.722 \\
\hline 20 & 0.44 & 0.046 & 0.147 & 0.198 & 0.255 & 0.594 & 0.632 & 0.662 & 0.716 \\
\hline 21 & 0.44 & 0.059 & 0.157 & 0.206 & 0.261 & 0.590 & 0.627 & 0.656 & 0.709 \\
\hline 22 & 0.44 & 0.070 & 0.165 & 0.213 & 0.266 & 0.586 & 0.622 & 0.651 & 0.704 \\
\hline 23 & 0.44 & 0.081 & 0.173 & 0.219 & 0.271 & 0.583 & 0.618 & 0.647 & 0.698 \\
\hline 24 & 0.44 & 0.091 & 0.181 & 0.225 & 0.276 & 0.579 & 0.614 & 0.642 & 0.693 \\
\hline 11 & 0.43 & -0.183 & -0.030 & 0.049 & 0.139 & 0.653 & 0.702 & 0.740 & 0.802 \\
\hline 12 & 0.43 & -0.142 & 0.002 & 0.075 & 0.159 & 0.641 & 0.688 & 0.725 & 0.787 \\
\hline 13 & 0.43 & -0.108 & 0.028 & 0.097 & 0.176 & 0.630 & 0.676 & 0.712 & 0.773 \\
\hline 14 & 0.43 & -0.079 & 0.050 & 0.115 & 0.190 & 0.622 & 0.666 & 0.701 & 0.761 \\
\hline 15 & 0.43 & -0.054 & 0.069 & 0.131 & 0.202 & 0.614 & 0.657 & 0.691 & 0.751 \\
\hline 16 & 0.43 & -0.032 & 0.086 & 0.145 & 0.212 & 0.607 & 0.649 & 0.683 & 0.741 \\
\hline 17 & 0.43 & -0.013 & 0.100 & 0.157 & 0.221 & 0.601 & 0.642 & 0.675 & 0.732 \\
\hline 18 & 0.43 & 0.004 & 0.113 & 0.168 & 0.230 & 0.595 & 0.635 & 0.667 & 0.724 \\
\hline 19 & 0.43 & 0.020 & 0.125 & 0.177 & 0.237 & 0.590 & 0.630 & 0.661 & 0.716 \\
\hline 20 & 0.43 & 0.034 & 0.135 & 0.186 & 0.243 & 0.586 & 0.624 & 0.655 & 0.710 \\
\hline 21 & 0.43 & 0.046 & 0.145 & 0.194 & 0.249 & 0.582 & 0.619 & 0.649 & 0.703 \\
\hline 22 & 0.43 & 0.058 & 0.153 & 0.201 & 0.255 & 0.578 & 0.615 & 0.644 & 0.697 \\
\hline 23 & 0.43 & 0.069 & 0.161 & 0.207 & 0.260 & 0.574 & 0.610 & 0.639 & 0.692 \\
\hline 24 & 0.43 & 0.078 & 0.169 & 0.213 & 0.264 & 0.571 & 0.606 & 0.635 & 0.686 \\
\hline 11 & 0.42 & -0.194 & -0.042 & 0.037 & 0.127 & 0.645 & 0.696 & 0.734 & 0.798 \\
\hline 12 & 0.42 & -0.154 & -0.010 & 0.063 & 0.147 & 0.633 & 0.682 & 0.719 & 0.782 \\
\hline 13 & 0.42 & -0.120 & 0.016 & 0.085 & 0.164 & 0.623 & 0.670 & 0.706 & 0.768 \\
\hline 14 & 0.42 & -0.091 & 0.038 & 0.103 & 0.178 & 0.614 & 0.659 & 0.695 & 0.756 \\
\hline 15 & 0.42 & -0.066 & 0.057 & 0.119 & 0.190 & 0.606 & 0.650 & 0.685 & 0.745 \\
\hline 16 & 0.42 & -0.045 & 0.074 & 0.133 & 0.201 & 0.599 & 0.642 & 0.676 & 0.735 \\
\hline 17 & 0.42 & -0.025 & 0.088 & 0.145 & 0.210 & 0.593 & 0.635 & 0.668 & 0.726 \\
\hline 18 & 0.42 & -0.008 & 0.101 & 0.156 & 0.218 & 0.587 & 0.628 & 0.661 & 0.718 \\
\hline 20 & 0.42 & 0.021 & 0.123 & 0.174 & 0.232 & 0.578 & 0.617 & 0.648 & 0.703 \\
\hline 21 & 0.42 & 0.034 & 0.133 & 0.182 & 0.238 & 0.574 & 0.612 & 0.642 & 0.697 \\
\hline 22 & 0.42 & 0.046 & 0.141 & 0.189 & 0.243 & 0.570 & 0.607 & 0.637 & 0.691 \\
\hline 23 & 0.42 & 0.056 & 0.149 & 0.196 & 0.248 & 0.566 & 0.603 & 0.632 & 0.685 \\
\hline
\end{tabular}


CONFIDENCE INTERVALS FOR KENDALL'S TAU

Table 1, continued.

\begin{tabular}{|c|c|c|c|c|c|c|c|c|c|}
\hline$N$ & Tau & L 99\% Cl & L 95\% Cl & $\mathrm{L} 90 \% \mathrm{Cl}$ & $\mathrm{L} 80 \% \mathrm{Cl}$ & $\mathrm{U} 80 \% \mathrm{Cl}$ & U $90 \% \mathrm{Ci}$ & U 95\% Cl & $\mathrm{U} 99 \% \mathrm{Cl}$ \\
\hline 24 & 0.42 & 0.066 & 0.157 & 0.202 & 0.253 & 0.563 & 0.599 & 0.628 & 0.680 \\
\hline 11 & 0.41 & -0.206 & -0.054 & 0.025 & 0.115 & 0.638 & 0.689 & 0.728 & 0.793 \\
\hline 12 & 0.41 & -0.166 & -0.022 & 0.051 & 0.136 & 0.626 & 0.675 & 0.713 & 0.777 \\
\hline 13 & 0.41 & -0.132 & 0.004 & 0.073 & 0.152 & 0.615 & 0.663 & 0.700 & 0.763 \\
\hline 14 & 0.41 & -0.103 & 0.026 & 0.091 & 0.166 & 0.606 & 0.652 & 0.689 & 0.751 \\
\hline 15 & 0.41 & -0.078 & 0.045 & 0.107 & 0.179 & 0.598 & 0.643 & 0.678 & 0.740 \\
\hline 16 & 0.41 & -0.057 & 0.062 & 0.121 & 0.189 & 0.591 & 0.635 & 0.669 & 0.730 \\
\hline 17 & 0.41 & -0.037 & 0.076 & 0.133 & 0.198 & 0.585 & 0.627 & 0.661 & 0.720 \\
\hline 18 & 0.41 & -0.020 & 0.089 & 0.144 & 0.206 & 0.580 & 0.621 & 0.654 & 0.712 \\
\hline 19 & 0.41 & -0.005 & 0.101 & 0.154 & 0.214 & 0.574 & 0.615 & 0.647 & 0.704 \\
\hline 20 & 0.41 & 0.009 & 0.111 & 0.162 & 0.220 & 0.570 & 0.609 & 0.641 & 0.697 \\
\hline 21 & 0.41 & 0.022 & 0.121 & 0.170 & 0.226 & 0.565 & 0.604 & 0.635 & 0.691 \\
\hline 22 & 0.41 & 0.034 & 0.129 & 0.177 & 0.232 & 0.562 & 0.599 & 0.630 & 0.685 \\
\hline 23 & 0.41 & 0.044 & 0.137 & 0.184 & 0.237 & 0.558 & 0.595 & 0.625 & 0.679 \\
\hline 24 & 0.41 & 0.054 & 0.145 & 0.190 & 0.242 & 0.554 & 0.591 & 0.620 & 0.673 \\
\hline 11 & 0.40 & -0.217 & -0.066 & 0.013 & 0.103 & 0.631 & 0.683 & 0.723 & 0.789 \\
\hline 12 & 0.40 & -0.177 & -0.034 & 0.039 & 0.124 & 0.619 & 0.669 & 0.707 & 0.773 \\
\hline 13 & 0.40 & -0.144 & -0.008 & 0.061 & 0.141 & 0.608 & 0.656 & 0.694 & 0.758 \\
\hline 14 & 0.40 & -0.115 & 0.014 & 0.080 & 0.155 & 0.599 & 0.645 & 0.682 & 0.746 \\
\hline 15 & 0.40 & -0.090 & 0.033 & 0.095 & 0.167 & 0.591 & 0.636 & 0.672 & 0.734 \\
\hline 16 & 0.40 & -0.069 & 0.050 & 0.109 & 0.177 & 0.584 & 0.628 & 0.663 & 0.724 \\
\hline 17 & 0.40 & -0.049 & 0.064 & 0.121 & 0.187 & 0.577 & 0.620 & 0.654 & 0.715 \\
\hline 18 & 0.40 & -0.032 & 0.077 & 0.132 & 0.195 & 0.572 & 0.613 & 0.647 & 0.706 \\
\hline 19 & 0.40 & -0.017 & 0.089 & 0.142 & 0.202 & 0.566 & 0.607 & 0.640 & 0.698 \\
\hline 20 & 0.40 & -0.003 & 0.099 & 0.151 & 0.209 & 0.562 & 0.602 & 0.634 & 0.691 \\
\hline 21 & 0.40 & 0.010 & 0.109 & 0.159 & 0.215 & 0.557 & 0.596 & 0.628 & 0.684 \\
\hline 22 & 0.40 & 0.022 & 0.118 & 0.166 & 0.221 & 0.553 & 0.591 & 0.622 & 0.678 \\
\hline 23 & 0.40 & 0.032 & 0.126 & 0.172 & 0.226 & 0.550 & 0.587 & 0.617 & 0.672 \\
\hline 24 & 0.40 & 0.042 & 0.133 & 0.179 & 0.230 & 0.546 & 0.583 & 0.613 & 0.667 \\
\hline 11 & -0.10 & -0.632 & -0.530 & -0.471 & -0.397 & 0.216 & 0.301 & 0.371 & 0.496 \\
\hline 12 & -0.10 & -0.606 & -0.507 & -0.450 & -0.380 & 0.196 & 0.277 & 0.343 & 0.464 \\
\hline 13 & -0.10 & -0.584 & -0.487 & -0.432 & -0.365 & 0.180 & 0.256 & 0.320 & 0.437 \\
\hline 14 & -0.10 & -0.565 & -0.470 & -0.417 & -0.352 & 0.166 & 0.239 & 0.300 & 0.413 \\
\hline 15 & -0.10 & -0.547 & -0.455 & -0.404 & -0.341 & 0.154 & 0.224 & 0.282 & 0.392 \\
\hline 16 & -0.10 & -0.532 & -0.442 & -0.392 & -0.332 & 0.143 & 0.210 & 0.267 & 0.373 \\
\hline 17 & -0.10 & -0.518 & -0.430 & -0.382 & -0.323 & 0.134 & 0.199 & 0.253 & 0.356 \\
\hline 18 & -0.10 & -0.505 & -0.419 & -0.372 & -0.315 & 0.125 & 0.188 & 0.241 & 0.341 \\
\hline 19 & -0.10 & -0.494 & -0.409 & -0.364 & -0.308 & 0.118 & 0.179 & 0.230 & 0.328 \\
\hline 20 & -0.10 & -0.483 & -0.401 & -0.356 & -0.302 & 0.111 & 0.170 & 0.220 & 0.315 \\
\hline 21 & -0.10 & -0.473 & -0.392 & -0.349 & -0.296 & 0.105 & 0.162 & 0.211 & 0.303 \\
\hline 22 & -0.10 & -0.464 & -0.385 & -0.342 & -0.291 & 0.099 & 0.155 & 0.202 & 0.293 \\
\hline 24 & -0.10 & -0.448 & -0.371 & -0.331 & -0.282 & 0.089 & 0.142 & 0.187 & 0.274 \\
\hline 11 & -0.11 & -0.638 & -0.537 & -0.479 & -0.406 & 0.206 & 0.292 & 0.362 & 0.489 \\
\hline 12 & -0.11 & -0.613 & -0.514 & -0.458 & -0.388 & 0.187 & 0.267 & 0.334 & 0.456 \\
\hline 13 & -0.11 & -0.591 & -0.495 & -0.441 & -0.374 & 0.170 & 0.247 & 0.311 & 0.429 \\
\hline
\end{tabular}


DAVID A. WALKER

Table 1, continued.

\begin{tabular}{|c|c|c|c|c|c|c|c|c|c|}
\hline$N$ & Tau & L 99\% Cl & L 95\% Cl & $\mathrm{L} 90 \% \mathrm{Cl}$ & $\mathrm{L} 80 \% \mathrm{Cl}$ & U $80 \% \mathrm{Cl}$ & U $90 \% \mathrm{Ci}$ & U 95\% Cl & U 99\% Cl \\
\hline 14 & -0.11 & -0.572 & -0.478 & -0.425 & -0.361 & 0.156 & 0.229 & 0.291 & 0.404 \\
\hline 15 & -0.11 & -0.554 & -0.463 & -0.412 & -0.350 & 0.144 & 0.214 & 0.273 & 0.383 \\
\hline 16 & -0.11 & -0.539 & -0.450 & -0.401 & -0.341 & 0.133 & 0.201 & 0.258 & 0.364 \\
\hline 17 & -0.11 & -0.525 & -0.438 & -0.390 & -0.332 & 0.124 & 0.189 & 0.244 & 0.347 \\
\hline 18 & -0.11 & -0.513 & -0.427 & -0.381 & -0.324 & 0.115 & 0.178 & 0.232 & 0.332 \\
\hline 19 & -0.11 & -0.501 & -0.418 & -0.372 & -0.318 & 0.108 & 0.169 & 0.220 & 0.318 \\
\hline 20 & -0.11 & -0.491 & -0.409 & -0.365 & -0.311 & 0.101 & 0.160 & 0.210 & 0.306 \\
\hline 21 & -0.11 & -0.481 & -0.401 & -0.358 & -0.306 & 0.094 & 0.152 & 0.201 & 0.294 \\
\hline 22 & -0.11 & -0.472 & -0.393 & -0.351 & -0.300 & 0.089 & 0.145 & 0.193 & 0.284 \\
\hline 23 & -0.11 & -0.463 & -0.387 & -0.345 & -0.295 & 0.083 & 0.138 & 0.185 & 0.274 \\
\hline 24 & -0.11 & -0.456 & -0.380 & -0.340 & -0.291 & 0.079 & 0.132 & 0.177 & 0.264 \\
\hline 11 & -0.12 & -0.644 & -0.544 & -0.487 & -0.414 & 0.197 & 0.283 & 0.353 & 0.481 \\
\hline 12 & -0.12 & -0.619 & -0.522 & -0.466 & -0.397 & 0.177 & 0.258 & 0.325 & 0.448 \\
\hline 13 & -0.12 & -0.597 & -0.502 & -0.449 & -0.382 & 0.160 & 0.237 & 0.302 & 0.420 \\
\hline 14 & -0.12 & -0.578 & -0.486 & -0.434 & -0.370 & 0.146 & 0.220 & 0.281 & 0.396 \\
\hline 15 & -0.12 & -0.561 & -0.471 & -0.421 & -0.359 & 0.134 & 0.204 & 0.264 & 0.375 \\
\hline 16 & -0.12 & -0.546 & -0.458 & -0.409 & -0.349 & 0.123 & 0.191 & 0.248 & 0.356 \\
\hline 17 & -0.12 & -0.532 & -0.446 & -0.399 & -0.341 & 0.114 & 0.179 & 0.234 & 0.339 \\
\hline 18 & -0.12 & -0.520 & -0.436 & -0.390 & -0.333 & 0.105 & 0.168 & 0.222 & 0.323 \\
\hline 19 & -0.12 & -0.509 & -0.426 & -0.381 & -0.327 & 0.098 & 0.159 & 0.211 & 0.309 \\
\hline 20 & -0.12 & -0.498 & -0.417 & -0.373 & -0.320 & 0.091 & 0.150 & 0.201 & 0.297 \\
\hline 21 & -0.12 & -0.489 & -0.409 & -0.366 & -0.315 & 0.084 & 0.142 & 0.191 & 0.285 \\
\hline 22 & -0.12 & -0.480 & -0.402 & -0.360 & -0.310 & 0.079 & 0.135 & 0.183 & 0.274 \\
\hline 23 & -0.12 & -0.471 & -0.395 & -0.354 & -0.305 & 0.073 & 0.128 & 0.175 & 0.264 \\
\hline 24 & -0.12 & -0.464 & -0.389 & -0.349 & -0.300 & 0.069 & 0.122 & 0.168 & 0.255 \\
\hline 11 & -0.13 & -0.650 & -0.551 & -0.494 & -0.422 & 0.187 & 0.273 & 0.344 & 0.473 \\
\hline 12 & -0.13 & -0.625 & -0.529 & -0.474 & -0.405 & 0.167 & 0.248 & 0.316 & 0.440 \\
\hline 13 & -0.13 & -0.604 & -0.510 & -0.457 & -0.391 & 0.150 & 0.228 & 0.292 & 0.412 \\
\hline 14 & -0.13 & -0.585 & -0.493 & -0.442 & -0.379 & 0.136 & 0.210 & 0.272 & 0.387 \\
\hline 15 & -0.13 & -0.568 & -0.479 & -0.429 & -0.368 & 0.124 & 0.195 & 0.254 & 0.366 \\
\hline 16 & -0.13 & -0.553 & -0.466 & -0.417 & -0.358 & 0.113 & 0.181 & 0.239 & 0.347 \\
\hline 17 & -0.13 & -0.540 & -0.454 & -0.407 & -0.350 & 0.104 & 0.169 & 0.225 & 0.330 \\
\hline 18 & -0.13 & -0.527 & -0.444 & -0.398 & -0.342 & 0.095 & 0.159 & 0.212 & 0.314 \\
\hline 19 & -0.13 & -0.516 & -0.434 & -0.390 & -0.336 & 0.088 & 0.149 & 0.201 & 0.300 \\
\hline 20 & -0.13 & -0.506 & -0.426 & -0.382 & -0.330 & 0.081 & 0.140 & 0.191 & 0.287 \\
\hline 21 & -0.13 & -0.496 & -0.418 & -0.375 & -0.324 & 0.074 & 0.132 & 0.181 & 0.276 \\
\hline 22 & -0.13 & -0.487 & -0.410 & -0.369 & -0.319 & 0.069 & 0.125 & 0.173 & 0.265 \\
\hline 23 & -0.13 & -0.479 & -0.404 & -0.363 & -0.314 & 0.063 & 0.118 & 0.165 & 0.255 \\
\hline 24 & -0.13 & -0.472 & -0.397 & -0.357 & -0.309 & 0.058 & 0.112 & 0.158 & 0.246 \\
\hline 11 & -0.14 & -0.656 & -0.558 & -0.502 & -0.431 & 0.177 & 0.264 & 0.335 & 0.465 \\
\hline 12 & -0.14 & -0.632 & -0.536 & -0.482 & -0.414 & 0.157 & 0.239 & 0.307 & 0.432 \\
\hline 14 & -0.14 & -0.592 & -0.501 & -0.450 & -0.387 & 0.126 & 0.200 & 0.263 & 0.379 \\
\hline 15 & -0.14 & -0.575 & -0.487 & -0.437 & -0.377 & 0.114 & 0.185 & 0.245 & 0.357 \\
\hline 16 & -0.14 & -0.560 & -0.474 & -0.426 & -0.367 & 0.103 & 0.171 & 0.229 & 0.338 \\
\hline 17 & -0.14 & -0.547 & -0.462 & -0.416 & -0.359 & 0.093 & 0.159 & 0.215 & 0.320 \\
\hline
\end{tabular}


CONFIDENCE INTERVALS FOR KENDALL'S TAU

Table 1, continued.

\begin{tabular}{|c|c|c|c|c|c|c|c|c|c|}
\hline$N$ & Tau & L 99\% Cl & L 95\% Cl & $\mathrm{L} 90 \% \mathrm{Cl}$ & $\mathrm{L} 80 \% \mathrm{Cl}$ & $\mathrm{U} 80 \% \mathrm{Cl}$ & U $90 \% \mathrm{Ci}$ & U 95\% Cl & $\mathrm{U} 99 \% \mathrm{Cl}$ \\
\hline 18 & -0.14 & -0.535 & -0.452 & -0.407 & -0.351 & 0.085 & 0.149 & 0.203 & 0.305 \\
\hline 19 & -0.14 & -0.524 & -0.443 & -0.398 & -0.345 & 0.077 & 0.139 & 0.191 & 0.291 \\
\hline 20 & -0.14 & -0.513 & -0.434 & -0.391 & -0.339 & 0.070 & 0.130 & 0.181 & 0.278 \\
\hline 21 & -0.14 & -0.504 & -0.426 & -0.384 & -0.333 & 0.064 & 0.122 & 0.172 & 0.266 \\
\hline 22 & -0.14 & -0.495 & -0.419 & -0.378 & -0.328 & 0.058 & 0.115 & 0.163 & 0.255 \\
\hline 23 & -0.14 & -0.487 & -0.412 & -0.372 & -0.323 & 0.053 & 0.108 & 0.155 & 0.245 \\
\hline 24 & -0.14 & -0.479 & -0.406 & -0.366 & -0.319 & 0.048 & 0.102 & 0.148 & 0.236 \\
\hline 11 & -0.15 & -0.662 & -0.565 & -0.510 & -0.439 & 0.167 & 0.254 & 0.326 & 0.457 \\
\hline 12 & -0.15 & -0.638 & -0.544 & -0.490 & -0.422 & 0.147 & 0.229 & 0.298 & 0.423 \\
\hline 13 & -0.15 & -0.617 & -0.525 & -0.473 & -0.408 & 0.130 & 0.208 & 0.274 & 0.395 \\
\hline 14 & -0.15 & -0.598 & -0.509 & -0.458 & -0.396 & 0.116 & 0.190 & 0.253 & 0.370 \\
\hline 15 & -0.15 & -0.582 & -0.494 & -0.445 & -0.385 & 0.104 & 0.175 & 0.235 & 0.348 \\
\hline 16 & -0.15 & -0.567 & -0.482 & -0.434 & -0.376 & 0.093 & 0.161 & 0.219 & 0.329 \\
\hline 17 & -0.15 & -0.554 & -0.470 & -0.424 & -0.368 & 0.083 & 0.149 & 0.205 & 0.311 \\
\hline 18 & -0.15 & -0.542 & -0.460 & -0.415 & -0.360 & 0.075 & 0.139 & 0.193 & 0.296 \\
\hline 19 & -0.15 & -0.531 & -0.451 & -0.407 & -0.354 & 0.067 & 0.129 & 0.181 & 0.281 \\
\hline 20 & -0.15 & -0.521 & -0.442 & -0.399 & -0.348 & 0.060 & 0.120 & 0.171 & 0.268 \\
\hline 21 & -0.15 & -0.512 & -0.434 & -0.393 & -0.342 & 0.054 & 0.112 & 0.162 & 0.257 \\
\hline 22 & -0.15 & -0.503 & -0.427 & -0.386 & -0.337 & 0.048 & 0.105 & 0.153 & 0.246 \\
\hline 23 & -0.15 & -0.495 & -0.421 & -0.380 & -0.332 & 0.043 & 0.098 & 0.145 & 0.236 \\
\hline 24 & -0.15 & -0.487 & -0.414 & -0.375 & -0.328 & 0.038 & 0.092 & 0.138 & 0.226 \\
\hline 11 & -0.16 & -0.667 & -0.572 & -0.517 & -0.447 & 0.157 & 0.245 & 0.317 & 0.449 \\
\hline 12 & -0.16 & -0.644 & -0.551 & -0.497 & -0.431 & 0.137 & 0.219 & 0.288 & 0.415 \\
\hline 13 & -0.16 & -0.623 & -0.532 & -0.481 & -0.416 & 0.120 & 0.198 & 0.264 & 0.386 \\
\hline 14 & -0.16 & -0.605 & -0.516 & -0.466 & -0.404 & 0.106 & 0.180 & 0.243 & 0.361 \\
\hline 15 & -0.16 & -0.589 & -0.502 & -0.454 & -0.394 & 0.093 & 0.165 & 0.225 & 0.339 \\
\hline 16 & -0.16 & -0.574 & -0.490 & -0.442 & -0.385 & 0.083 & 0.151 & 0.209 & 0.319 \\
\hline 17 & -0.16 & -0.561 & -0.478 & -0.433 & -0.377 & 0.073 & 0.139 & 0.195 & 0.302 \\
\hline 18 & -0.16 & -0.549 & -0.468 & -0.424 & -0.369 & 0.065 & 0.129 & 0.183 & 0.286 \\
\hline 19 & -0.16 & -0.538 & -0.459 & -0.415 & -0.363 & 0.057 & 0.119 & 0.171 & 0.272 \\
\hline 20 & -0.16 & -0.528 & -0.450 & -0.408 & -0.357 & 0.050 & 0.110 & 0.161 & 0.259 \\
\hline 21 & -0.16 & -0.519 & -0.443 & -0.401 & -0.351 & 0.044 & 0.102 & 0.152 & 0.247 \\
\hline 22 & -0.16 & -0.510 & -0.436 & -0.395 & -0.346 & 0.038 & 0.095 & 0.143 & 0.236 \\
\hline 23 & -0.16 & -0.503 & -0.429 & -0.389 & -0.341 & 0.033 & 0.088 & 0.135 & 0.226 \\
\hline 24 & -0.16 & -0.495 & -0.423 & -0.384 & -0.337 & 0.028 & 0.082 & 0.128 & 0.217 \\
\hline 11 & -0.17 & -0.673 & -0.579 & -0.525 & -0.455 & 0.147 & 0.235 & 0.308 & 0.441 \\
\hline 12 & -0.17 & -0.650 & -0.558 & -0.505 & -0.439 & 0.127 & 0.210 & 0.279 & 0.406 \\
\hline 13 & -0.17 & -0.629 & -0.540 & -0.489 & -0.425 & 0.110 & 0.189 & 0.255 & 0.377 \\
\hline 14 & -0.17 & -0.611 & -0.524 & -0.474 & -0.413 & 0.096 & 0.171 & 0.234 & 0.352 \\
\hline 15 & -0.17 & -0.595 & -0.510 & -0.462 & -0.403 & 0.083 & 0.155 & 0.216 & 0.330 \\
\hline 16 & -0.17 & -0.581 & -0.497 & -0.451 & -0.393 & 0.072 & 0.141 & 0.200 & 0.310 \\
\hline 18 & -0.17 & -0.556 & -0.476 & -0.432 & -0.378 & 0.054 & 0.118 & 0.173 & 0.277 \\
\hline 19 & -0.17 & -0.546 & -0.467 & -0.424 & -0.371 & 0.047 & 0.109 & 0.161 & 0.262 \\
\hline 20 & -0.17 & -0.536 & -0.459 & -0.417 & -0.365 & 0.040 & 0.100 & 0.151 & 0.249 \\
\hline 21 & -0.17 & -0.527 & -0.451 & -0.410 & -0.360 & 0.034 & 0.092 & 0.142 & 0.237 \\
\hline
\end{tabular}


DAVID A. WALKER

Table 1, continued.

\begin{tabular}{|c|c|c|c|c|c|c|c|c|c|}
\hline$N$ & Tau & L 99\% Cl & L 95\% Cl & $\mathrm{L} 90 \% \mathrm{Cl}$ & $\mathrm{L} 80 \% \mathrm{Cl}$ & U $80 \% \mathrm{Cl}$ & U $90 \% \mathrm{Ci}$ & U 95\% Cl & U $99 \% \mathrm{Cl}$ \\
\hline 22 & -0.17 & -0.518 & -0.444 & -0.404 & -0.355 & 0.028 & 0.084 & 0.133 & 0.226 \\
\hline 23 & -0.17 & -0.510 & -0.437 & -0.398 & -0.350 & 0.022 & 0.078 & 0.125 & 0.216 \\
\hline 24 & -0.17 & -0.503 & -0.431 & -0.393 & -0.346 & 0.018 & 0.071 & 0.118 & 0.207 \\
\hline 11 & -0.18 & -0.679 & -0.586 & -0.532 & -0.464 & 0.137 & 0.225 & 0.298 & 0.432 \\
\hline 12 & -0.18 & -0.656 & -0.565 & -0.513 & -0.447 & 0.117 & 0.200 & 0.269 & 0.398 \\
\hline 13 & -0.18 & -0.635 & -0.547 & -0.496 & -0.433 & 0.100 & 0.179 & 0.245 & 0.368 \\
\hline 14 & -0.18 & -0.618 & -0.531 & -0.482 & -0.422 & 0.085 & 0.160 & 0.224 & 0.343 \\
\hline 15 & -0.18 & -0.602 & -0.517 & -0.470 & -0.411 & 0.073 & 0.145 & 0.206 & 0.321 \\
\hline 16 & -0.18 & -0.588 & -0.505 & -0.459 & -0.402 & 0.062 & 0.131 & 0.190 & 0.301 \\
\hline 17 & -0.18 & -0.575 & -0.494 & -0.449 & -0.394 & 0.053 & 0.119 & 0.176 & 0.283 \\
\hline 18 & -0.18 & -0.563 & -0.484 & -0.440 & -0.387 & 0.044 & 0.108 & 0.163 & 0.267 \\
\hline 19 & -0.18 & -0.553 & -0.475 & -0.432 & -0.380 & 0.036 & 0.098 & 0.151 & 0.253 \\
\hline 20 & -0.18 & -0.543 & -0.467 & -0.425 & -0.374 & 0.030 & 0.090 & 0.141 & 0.240 \\
\hline 21 & -0.18 & -0.534 & -0.459 & -0.418 & -0.369 & 0.023 & 0.082 & 0.131 & 0.228 \\
\hline 22 & -0.18 & -0.526 & -0.452 & -0.412 & -0.364 & 0.017 & 0.074 & 0.123 & 0.217 \\
\hline 23 & -0.18 & -0.518 & -0.446 & -0.407 & -0.359 & 0.012 & 0.067 & 0.115 & 0.206 \\
\hline 24 & -0.18 & -0.510 & -0.440 & -0.401 & -0.355 & 0.007 & 0.061 & 0.107 & 0.197 \\
\hline 11 & -0.19 & -0.684 & -0.593 & -0.539 & -0.472 & 0.127 & 0.215 & 0.289 & 0.424 \\
\hline 12 & -0.19 & -0.661 & -0.572 & -0.520 & -0.455 & 0.106 & 0.190 & 0.260 & 0.389 \\
\hline 13 & -0.19 & -0.642 & -0.554 & -0.504 & -0.442 & 0.089 & 0.169 & 0.235 & 0.359 \\
\hline 14 & -0.19 & -0.624 & -0.539 & -0.490 & -0.430 & 0.075 & 0.150 & 0.214 & 0.334 \\
\hline 15 & -0.19 & -0.609 & -0.525 & -0.478 & -0.420 & 0.063 & 0.135 & 0.196 & 0.311 \\
\hline 16 & -0.19 & -0.595 & -0.513 & -0.467 & -0.411 & 0.052 & 0.121 & 0.180 & 0.291 \\
\hline 17 & -0.19 & -0.582 & -0.502 & -0.457 & -0.403 & 0.042 & 0.109 & 0.165 & 0.274 \\
\hline 18 & -0.19 & -0.570 & -0.492 & -0.449 & -0.396 & 0.034 & 0.098 & 0.153 & 0.258 \\
\hline 19 & -0.19 & -0.560 & -0.483 & -0.441 & -0.389 & 0.026 & 0.088 & 0.141 & 0.243 \\
\hline 20 & -0.19 & -0.550 & -0.475 & -0.433 & -0.383 & 0.019 & 0.079 & 0.131 & 0.230 \\
\hline 21 & -0.19 & -0.541 & -0.467 & -0.427 & -0.378 & 0.013 & 0.071 & 0.121 & 0.218 \\
\hline 22 & -0.19 & -0.533 & -0.460 & -0.421 & -0.373 & 0.007 & 0.064 & 0.113 & 0.207 \\
\hline 23 & -0.19 & -0.525 & -0.454 & -0.415 & -0.368 & 0.002 & 0.057 & 0.105 & 0.196 \\
\hline 24 & -0.19 & -0.518 & -0.448 & -0.410 & -0.364 & -0.003 & 0.051 & 0.097 & 0.187 \\
\hline 11 & -0.20 & -0.690 & -0.600 & -0.547 & -0.480 & 0.117 & 0.205 & 0.279 & 0.415 \\
\hline 12 & -0.20 & -0.667 & -0.579 & -0.528 & -0.464 & 0.096 & 0.180 & 0.250 & 0.380 \\
\hline 13 & -0.20 & -0.648 & -0.561 & -0.512 & -0.450 & 0.079 & 0.158 & 0.225 & 0.350 \\
\hline 14 & -0.20 & -0.630 & -0.546 & -0.498 & -0.438 & 0.065 & 0.140 & 0.204 & 0.324 \\
\hline 15 & -0.20 & -0.615 & -0.532 & -0.486 & -0.428 & 0.052 & 0.124 & 0.186 & 0.302 \\
\hline 16 & -0.20 & -0.601 & -0.520 & -0.475 & -0.419 & 0.042 & 0.111 & 0.170 & 0.282 \\
\hline 17 & -0.20 & -0.589 & -0.510 & -0.466 & -0.411 & 0.032 & 0.099 & 0.155 & 0.264 \\
\hline 18 & -0.20 & -0.577 & -0.500 & -0.457 & -0.404 & 0.023 & 0.088 & 0.143 & 0.248 \\
\hline 19 & -0.20 & -0.567 & -0.491 & -0.449 & -0.398 & 0.016 & 0.078 & 0.131 & 0.233 \\
\hline 20 & -0.20 & -0.557 & -0.483 & -0.442 & -0.392 & 0.009 & 0.069 & 0.121 & 0.220 \\
\hline 22 & -0.20 & -0.540 & -0.468 & -0.429 & -0.382 & -0.003 & 0.054 & 0.102 & 0.197 \\
\hline 23 & -0.20 & -0.533 & -0.462 & -0.424 & -0.377 & -0.009 & 0.047 & 0.094 & 0.186 \\
\hline 24 & -0.20 & -0.526 & -0.456 & -0.419 & -0.373 & -0.014 & 0.040 & 0.087 & 0.177 \\
\hline
\end{tabular}

881 


\section{CONFIDENCE INTERVALS FOR KENDALL'S TAU}

\section{References}

American Psychological Association. (2010). Publication manual of the American Psychological Association (6th ed.). Washington, DC: American Psychological Association.

Bonett, D. G., \& Wright, T. A. (2000). Sample size requirements for estimating Pearson, Kendall and Spearman correlations. Psychometrika, 65(1), 23-28. doi: 10.1007/BF02294183

Cohen, J. (1990). Things I have learned (so far). American Psychologist, 45(1), 1304-1312. doi: 10.1037/0003-066X.45.12.1304

Cooper, H., \& Hedges, L. V. (Eds.). (1994). The handbook of research synthesis. New York, NY: Russell Sage Foundation.

Fieller, E. C., Hartley, H. O., \& Pearson, E. S. (1957). Tests for rank correlation coefficients. Biometrika, 44(3/4), 470-481. doi: 10.2307/2332878

Fisher, R. A. (1925). Statistical methods for research workers. London: Hafner Press.

Gilpin, A. R. (1993). Table for conversion of Kendall's tau to Spearman's rho within the context of measures of magnitude of effect for meta-analysis. Educational and Psychological Measurement, 53(1), 87-92. doi:

10.1177/0013164493053001007

Helsel, D. R., \& Hirsch, R. M. (1995). Statistical methods in water resources. Amsterdam: Elsevier Science.

Kendall, M. G. (1949). Rank and product-moment correlation. Biometrika, 36(1/2), 177-193. doi: 10.2307/2332540

Levin, J. R., \& Robinson, D. H. (2003). The trouble with interpreting statistically nonsignificant effect sizes in single-study investigations. Journal of Modern Applied Statistical Methods, 2(1), 231-236. Retrieved from http://digitalcommons.wayne.edu/jmasm/vol2/iss1/23/

Long, J. D., \& Cliff, N. (1997). Confidence intervals for Kendall's tau. British Journal of Mathematical and Statistical Psychology, 50(1), 31-41. doi: 10.1111/j.2044-8317.1997.tb01100.x

Rupinski, M. T., \& Dunlap, W. P. (1996). Approximating Pearson productmoment correlations from Kendall's tau and Spearman's rho. Educational and Psychological Measurement, 56(3), 419-429. doi:

10.1177/0013164496056003004 


\section{DAVID A. WALKER}

Tukey, J. W. (1960). Conclusions vs decisions. Technometrics, 2(4), 423433. doi: 10.1080/00401706.1960.10489909

Walker, D. A. (2015). Confidence intervals for Kendall's tau with small samples. [Computer program]. DeKalb, IL: Author. 